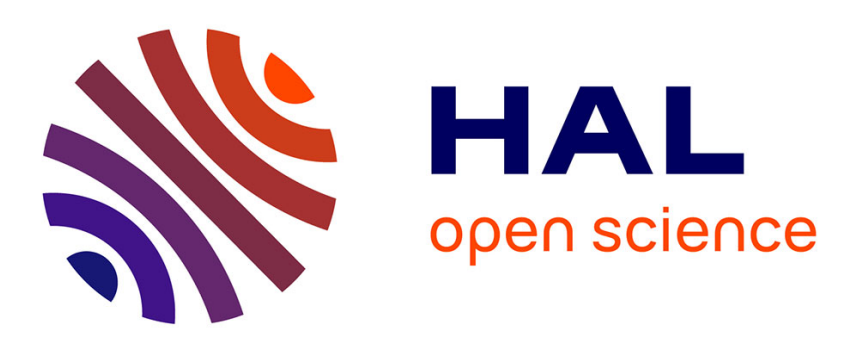

\title{
Fast and Accurate Computation of Orbital Collision Probability for Short-Term Encounters
}

Romain Serra, Denis Arzelier, Mioara Joldes, Jean-Bernard Lasserre, Aude Rondepierre, Bruno Salvy

\section{- To cite this version:}

Romain Serra, Denis Arzelier, Mioara Joldes, Jean-Bernard Lasserre, Aude Rondepierre, et al.. Fast and Accurate Computation of Orbital Collision Probability for Short-Term Encounters. Journal of Guidance, Control, and Dynamics, 2016, 39 (5), pp.1009-1021. 10.2514/1.G001353 . hal-01132149

HAL Id: hal-01132149

https://hal.science/hal-01132149

Submitted on 16 Mar 2015

HAL is a multi-disciplinary open access archive for the deposit and dissemination of scientific research documents, whether they are published or not. The documents may come from teaching and research institutions in France or abroad, or from public or private research centers.
L'archive ouverte pluridisciplinaire HAL, est destinée au dépôt et à la diffusion de documents scientifiques de niveau recherche, publiés ou non, émanant des établissements d'enseignement et de recherche français ou étrangers, des laboratoires publics ou privés. 


\title{
A Fast and Accurate Method to Compute the
}

\section{Probability of Collision for Short-term Space}

\section{Encounters}

\author{
Romain Serra $^{1}$, Denis Arzelier ${ }^{2}$, Mioara Joldes ${ }^{3}$, Jean-Bernard Lasserre ${ }^{4}$ \\ CNRS ; LAAS ; 7 avenue du colonel Roche, F-31077 Toulouse, France \\ Université de Toulouse ; UPS, INSA, INP, ISAE ; LAAS ; F-31077 Toulouse, France \\ Aude Rondepierre ${ }^{5}$ \\ Institut de Mathématiques de Toulouse, \\ Université de Toulouse; INSA; F-31062 Toulouse, France \\ Bruno Salvy ${ }^{6}$ \\ INRIA; ENS; 69000 Lyon, France
}

This article provides a new method for computing the probability of collision between two spherical space objects involved in a short-term encounter under Gaussiandistributed uncertainty. In this model of conjunction, classical assumptions reduce the probability of collision to the integral of a two-dimensional Gaussian probability density function over a disk. The computational method presented here is based on an analytic expression for the integral, derived by use of Laplace transform and D-finite functions properties. The formula has the form of a product between an exponential term and a convergent power series with positive coefficients. Analytic bounds on the truncation error are also derived and are used to obtain a very accurate algorithm.

\footnotetext{
$1 \mathrm{Ph}$. D. Student CNRS, LAAS-CNRS, Methods and Algorithms in Control, serra@laas.fr 2 Senior Researcher CNRS, LAAS-CNRS, Methods and Algorithms in Control, arzelier@laas.fr 3 Researcher CNRS, LAAS-CNRS, Methods and Algorithms in Control, mjoldes@laas.fr 4 Senior Researcher CNRS, LAAS-CNRS, Methods and Algorithms in Control, lasserre@laas.fr 5 Assistant Professor, IMT, aude.rondepierre@math.univ-toulouse.fr 6 Senior Researcher, INRIA, LIP-ENS Lyon, bruno.salvy@inria.fr
} 
Another contribution is the derivation of analytic bounds on the probability of collision itself, allowing for a very fast and - in most cases - very precise evaluation of the risk. The only other analytical method of the literature - based on an approximation - is shown to be a special case of the new formula. A numerical study illustrates the efficiency of the proposed algorithms on a broad variety of examples and favorably compares the approach to the other methods of the literature.

\section{Nomenclature}

$\begin{array}{ll}\mathcal{P}_{c} & \text { Probability of collision } \\ \mathbf{1}_{A} & \text { Indicator function for set } A: \mathbf{1}_{A}(x)=1 \text { if } x \in A \text { else } \mathbf{1}_{A}(x)=0 \\ \mathcal{L}_{f} & \text { Laplace transform of function } f \\ \overline{\mathcal{B}}(M, \delta) & \text { Closed disk of center } M \text { and radius } \delta \\ \operatorname{Re}(\lambda) & \text { Real part of complex number } \lambda \\ R & \text { Combined sphere radius, m } \\ \mu(r) & \text { Mean of the random vector } r \\ \Sigma & \text { Covariance matrix } \\ \sigma & \text { Standard deviation, m } \\ \lceil\cdot 7 & \text { the ceiling function which maps a real number to the smallest following integer. } \\ \text { Subscript } & \\ p & \text { Primary object } \\ s & \text { Secondary object } \\ m & \text { Mean value }\end{array}$

\section{Introduction}

The number of space debris has been drastically increasing during the last decades, becoming an issue for on-orbit safety. These debris can indeed cause serious damage to operational Earth- 
orbiting spacecraft. Avoiding collision has become a usual and necessary task for many satellites on duty. In order to prevent collisions, space debris are nowadays radar-tracked and conjunctions with operational spacecraft can be predicted. From this information on a possible collision between two objects, it is then the task of the operator to assess the risk. Because of the uncertain nature of the data, the decision parameter is often computed as a probability of collision. If it exceeds some tolerance threshold, an evasive maneuver will be performed. When modeling conjunctions, two assumptions are usually made in the literature [1-6]. First, the geometrical shape of the two objects is supposed to be spherical. Second, uncertainty on the state vectors of the two objects is modeled with independent normal probability distributions.

Moreover, in this article, only short-term encounters are considered i.e., conjunctions with high relative velocities. Such cases typically arise in Low Earth Orbit where orbital velocities are fairly high. Two assumptions define the short-term encounter model [2-6]. First, the relative trajectory of the objects during the encounter is considered to be a straight line $[5,6]$. Secondly, uncertainty on the velocities is neglected. The probability of collision can then be expressed as a two-dimensional Gaussian integral over a disk. The integrand is the probability density function of the relative coordinates in the so-called encounter plane [5] while the domain of integration is the cross-section of the spherical combined object. As a result, the only inputs needed for the computation of the probability of collision are: the radius of the combined spherical object, the miss distance and the covariance matrix of the relative position in the encounter plane at reference time. A more detailed description is given in Section II.

The computation of the short-term encounter probability of collision has been dealt with in several ways. First, as a probability, it can be obtained from Monte Carlo trials [7], but this is a time-consuming process. Another class of methods are based on numerical integration schemes. In chronological order of apparition, they are: Foster's [8], Patera's [3] and Alfano's [9]. The main disadvantage of this family of methods is that they are strongly dependent upon the chosen integration method and suppose to manage a sensitive trade-off between precision and computation time. Finally, the approach closest to ours is Chan's [5, 10] who derives an analytic formula, but with an approximation with respect to the initial model. 


\section{Contribution}

In Section III a new analytical formula is proposed, with the following distinguishing features:

- It has the form of a product between an exponential term and a convergent power series with positive terms. The series is obtained by use of Laplace transform techniques [11]. The explicit form of the linear recurrence satisfied by the coefficients of the series is derived using properties of D-finite functions i.e., solutions of linear ordinary differential equations with polynomial coefficients $[12,13]$.

- Analytical bounds on the truncation error are provided, enabling the user to evaluate the truncated series as accurately as required. As a by-product of these bounds on the truncation error, one obtains analytical bounds on the probability of collision.

- In contrast to Chan's method, it does not make any approximation of the integral to be computed. In is shown in Section IV that Chan's formula is a special case of the new formula.

- When compared to other methods on a large number of test cases, the resulting algorithm performs well in practice. As illustrated in Section V by numerical examples, it allows for a very accurate and fast way to estimate the risk in most cases, which is highly desirable from an operator point of view.

\section{Encounter Model}

In this section, the general framework of on-orbit collision risk is briefly described. Next, we present the model of short-term encounters between two spherical objects under Gaussiandistributed uncertainty.

\section{A. General encounter between two space objects}

The system considered is composed of two space objects subject to orbital dynamics over some time interval. One is called the primary object $p$ and the other one the secondary object $s$. Typically, the primary is an active spacecraft while the secondary is a space debris. Information - such as radar measurements - describes their respective geometry, position and velocity at some detection time. 
The initial conditions - position and velocity of $p$ and $s$ at reference time - are supposed to be uncertain parameters, resulting in uncertain dynamics for each object. The reference time usually belongs to the time interval when both objects are in the collision range. Generally, it is the so-called nominal time of closest approach (TCA) [2]. This date is defined as the instant when the mean relative distance between the two objects reaches its global minimum over time. This minimum value is another quantity of great use referred to as the nominal miss distance [2]. Considering that statistical assumptions are usually made on these initial conditions, the collision risk is naturally characterized via a probabilistic metric. As a result, it can be defined as the integral of a probability density function (pdf) over some domain of integration. Without any further assumption, computing the probability of collision is a hard integration problem for two main reasons. First, the domain of integration is often impossible to derive analytically, because of its dependency upon the dynamics of each object which may be fairly complex depending on the model being considered. Secondly, integrating the probability density function over this domain is also difficult in general since, even for instance with Gaussian density functions, such an integral generally admits no closed-form formula [14].

A general method to handle the computation of the probability of collision is based on MonteCarlo trials [7]. It consists in simulating the dynamics of a large number of scenarios and in counting the number of effective collisions. Unfortunately, the number of required trials goes up with the targeted precision and makes this method quite time-consuming. For so-called rare events - events with very low probability values - it can even become intractable [7]. Therefore, simplified encounter models have been designed to reduce the complexity of the computation of the probability of collision, allowing for faster dedicated algorithms.

\section{B. Short-term encounter between spherical objects}

One of these simplified encounter models is the so-called short-term encounter model $[2,5,6,8]$ that can be used to describe a number of conjunctions when the magnitude of relative velocities at stake is sufficiently high. This typically happens in Low-Earth Orbit (LEO) where orbital velocities are the largest in norm [5]. Investigations about the range of validity of the short-term encounter 
model can be found in $[5,15]$. In the case of a short-term encounter between spherical objects under Gaussian-distributed uncertainty, the probability of collision reduces to a 2-D Gaussian integral over a disk $[2,5,6,8]$. The present article addresses the practical computation of this quantity. Five assumptions are needed to define the short-term encounter model under Gaussian-distributed uncertainty for spherical objects:

1. The relative trajectories are approximated as rectilinear.

2. The velocities at reference time are considered as deterministic variables.

3. Initial position vectors of both objects are Gaussian independent random vectors.

4. Each object is approximated by a spherical geometrical shape.

5. The time boundaries of the conjunction are extended to infinity.

\section{Encounter frame}

The fact that the relative motion is rectilinear motivates the choice of a frame of study with one axis along the relative velocity. One possibility is to introduce the so-called encounter frame $[2,3,5,8]$ defined at reference time. Its exact definition depends on the authors (see for instance [2] and [5] for two different frames) but it always has the two following characteristics: it is centered on the mean position of one of the two objects and is built from the so-called encounter plane. This plane contains the origin and is orthogonal to the direction of the relative velocity.

The configuration considered in this study is represented in Figure 1. The origin of the frame is located at the center of the primary object $p$. The basis vector $e_{\tilde{z}}$ is oriented along the relative velocity $v=v_{s}-v_{p}$. The basis vector $e_{\tilde{x}}$ belongs to the encounter plane: it points towards the orthogonal projection of the mean relative position $\mu\left(r^{0}\right)$ onto the encounter plane. Finally, the basis vector $e_{\tilde{y}}$ completes the right-handed system and thus belongs to the encounter plane as well. In summary, one has

$$
e_{\tilde{z}}=\frac{v}{\|v\|}, \quad e_{\tilde{y}}=\frac{v \times \mu\left(r^{0}\right)}{\left\|v \times \mu\left(r^{0}\right)\right\|}, e_{\tilde{x}}=e_{\tilde{y}} \times e_{\tilde{z}}
$$

Let $\left(\tilde{x}_{m}, 0, \tilde{z}_{m}\right)$ be the coordinates of the mean relative position in the encounter frame where 
the $\tilde{y}$ coordinate is zero by construction. It is worth noticing that, since the relative trajectory is rectilinear, $\tilde{x}_{m}$ is in fact equal to the miss distance.

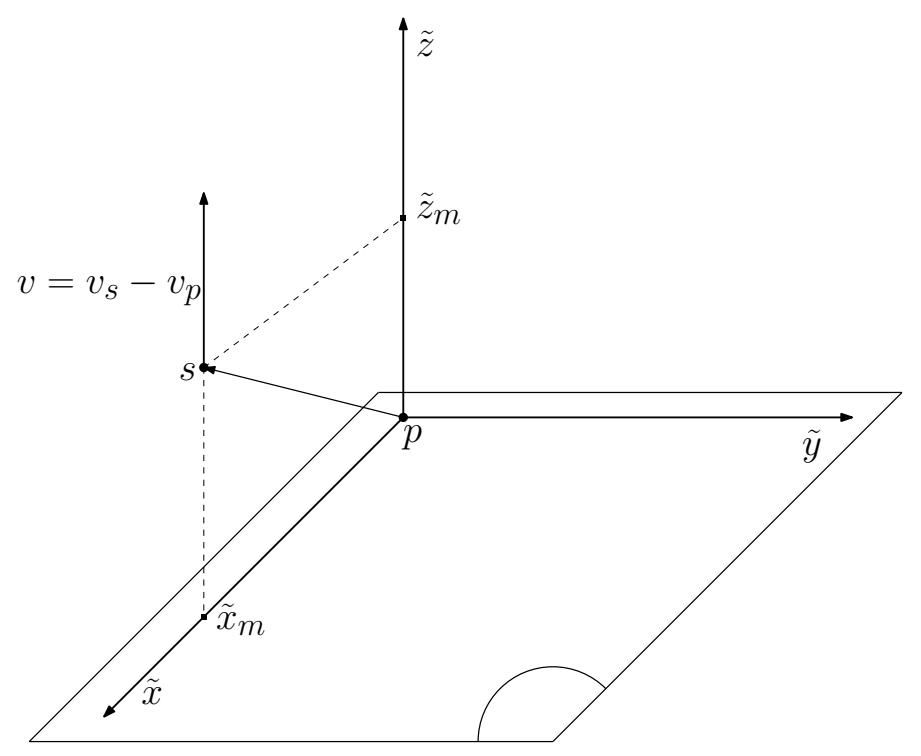

Figure 1: Encounter plane and frame $\left(e_{\tilde{x}}, e_{\tilde{y}}, e_{\tilde{z}}\right)$

\section{Integral representation}

Under the assumptions of the short-term encounter model, the probability of collision can be formulated as a 2-D integral in the encounter plane. For spherical objects, the domain of integration is a closed disk $\overline{\mathcal{B}}((0,0), R)$ centered at the origin of radius $R$. The quantity $R$ is the combined radius and is defined as the sum of the respective radii of the two objects i.e. $R=R_{p}+R_{s}$. The two-dimensional pdf involved in the probability of collision describes the distribution of the relative position in the encounter plane. From the hypothesis on the nature of uncertainty, it is a multivariate normal law. Therefore, it is completely defined by its mean vector and its variancecovariance matrix [16]. Let $\Sigma_{\tilde{x} \tilde{y}}$ be the covariance matrix of the relative coordinates in the encounter plane. The probability of collision can then be written as:

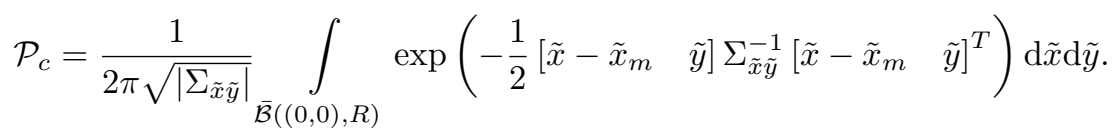

Equation (2) shows that the probability of collision only depends on the combined radius $R$, the miss distance $\tilde{x}_{m}$ and the covariance matrix $\Sigma_{\tilde{x} \tilde{y}}$ of the relative coordinates in the encounter plane. 


\section{Frame rotation}

Further calculations allow to write a formula with a simpler integrand. As a covariance matrix, $\Sigma_{\tilde{x} \tilde{y}}$ can be written as:

$$
\Sigma_{\tilde{x} \tilde{y}}=\left[\begin{array}{cc}
\sigma_{\tilde{x}}^{2} & \rho_{\tilde{x} \tilde{y}} \sigma_{\tilde{x}} \sigma_{\tilde{y}} \\
\rho_{\tilde{x} \tilde{y}} \sigma_{\tilde{x}} \sigma_{\tilde{y}} & \sigma_{\tilde{y}}^{2}
\end{array}\right]
$$

where $\sigma_{\tilde{x}}, \sigma_{\tilde{y}} \in \mathbb{R}_{*}^{+}$are the standard deviations of the relative coordinates in the encounter plane and $\rho_{\tilde{x} \tilde{y}} \in(-1,1)$ is the correlation coefficient. In order to eliminate the cross-terms of the Gaussian function, a rotation to the principal axis of the covariance matrix is performed in the encounter plane (see Figure 2). The new coordinates, denoted $(x, y)$, are respectively along the major and the minor axis. Consistently with Chan's notation [5], let $-\theta$ be the corresponding rotation angle. Nevertheless, the reader should be aware that Chan actually uses this symbol to denote any angle rotating to the principal axis, not necessarily with the major axis aligned with the first axis as done here. This transformation does not change the nature of the domain of integration which remains a disk of radius $R$ centered at the origin. It follows that:

$$
\mathcal{P}_{c}=\frac{1}{2 \pi \sigma_{x} \sigma_{y}} \int_{\overline{\mathcal{B}}((0,0), R)} \exp \left(-\frac{1}{2}\left(\frac{\left(x-x_{m}\right)^{2}}{\sigma_{x}^{2}}+\frac{\left(y-y_{m}\right)^{2}}{\sigma_{y}^{2}}\right)\right) \mathrm{d} x \mathrm{~d} y
$$

where the quantities $\sigma_{x}$ and $\sigma_{y}$ are standard deviations of the new coordinates. As a matter of fact, $\sigma_{x}^{2}$ and $\sigma_{y}^{2}$ are respectively the largest and the smallest eigenvalues of $\Sigma_{\tilde{x} \tilde{y}}$ :

$$
\left\{\frac{\sigma_{\tilde{x}}^{2}+\sigma_{\tilde{y}}^{2}}{2} \pm \sqrt{\left(\frac{\sigma_{\tilde{x}}^{2}-\sigma_{\tilde{y}}^{2}}{2}\right)^{2}+\rho_{\tilde{x} \tilde{y}}^{2} \sigma_{\tilde{x}}^{2} \sigma_{\tilde{y}}^{2}}\right\}
$$

Accordingly to the rotation of angle $-\theta$, one has:

$$
x_{m}=\tilde{x}_{m} \cos \theta, y_{m}=-\tilde{x}_{m} \sin \theta
$$

The angle $\theta$ can always be chosen in $\left(-\frac{\pi}{2}, \frac{\pi}{2}\right]$. If $\rho_{\tilde{x} \tilde{y}} \neq 0$, it is given by the following formula:

$$
\theta=\arctan \left(\frac{\sigma_{\tilde{y}}^{2}-\sigma_{\tilde{x}}^{2}}{2 \rho_{\tilde{x} \tilde{y}} \sigma_{\tilde{x}} \sigma_{\tilde{y}}}+\operatorname{sign}\left(\rho_{\tilde{x} \tilde{y}}\right) \sqrt{1+\left(\frac{\sigma_{\tilde{y}}^{2}-\sigma_{\tilde{x}}^{2}}{2 \rho_{\tilde{x} \tilde{y}} \sigma_{\tilde{x}} \sigma_{\tilde{y}}}\right)^{2}}\right)
$$

If $\rho_{\tilde{x} \tilde{y}}=0$, the matrix $\Sigma_{\tilde{x} \tilde{y}}$ is in fact already diagonal and two subcases can occur. If $\sigma_{\tilde{x}} \geq \sigma_{\tilde{y}}$, no rotation is needed and $\theta=0$; if $\sigma_{\tilde{x}}<\sigma_{\tilde{y}}$, one only needs to interchange the axis and $\theta=\frac{\pi}{2}$. 


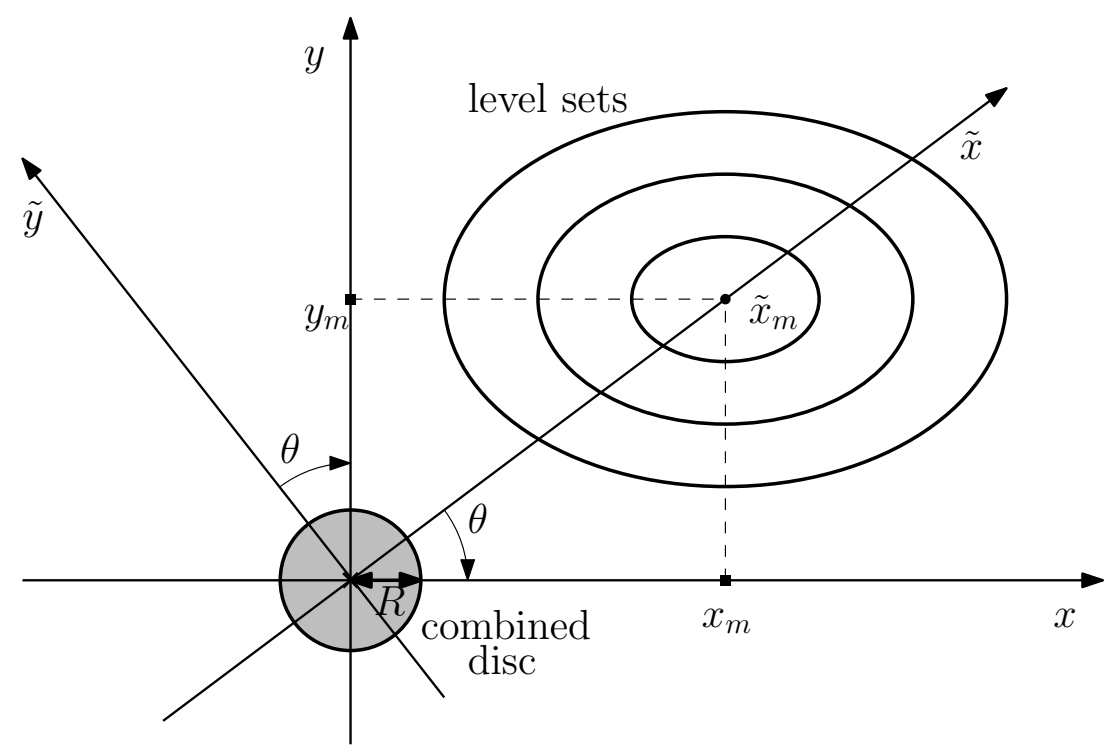

Figure 2: Rotation to the principal axis of the covariance matrix in the encounter plane

Equation (4) defines the so-called short-encounter formula for the probability of collision between two spherical objects under Gaussian-distributed uncertainty. It has the form of a 2-D integral over a disk centered at the origin of a Gaussian function with no cross-terms. Several methods have been developed in the literature to compute this integral and are summarized in the following subsection. The contribution of the article is to propose a new systematic computation method that will be presented in details in the next section.

\section{State of the art for the computation of $\mathcal{P}_{c}$}

Monte Carlo methods can be used to compute the probability of collision for short-term encounters. But as for the general collision model, they are time-consuming and ill-fitted to compute probabilities with very low values. Moreover, they do not take advantage of the particular expression of the short-term encounter formula. On the other hand, four methods have been developed so far to specifically handle this computation.

The first of these methods - used by NASA [5] - originates from an article by Foster and Estes [8]. It consists in a direct application of a numerical discretization scheme to the two-dimensional integral after switching to polar coordinates. While being fairly precise, it is relatively slow compared to other methods [5]. Patera [3] reformulates the probability of collision as a one-dimensional integral 
by writing it as a path-integral over the contour of the domain of integration. A numerical scheme - namely the midpoint method - is used for actual computation. By use of the error function, Alfano [4] also formulates the probability of collision as a one-dimensional integral. Then, Simpson's integration scheme is applied to compute it. The outcome is a finite sum involving exponential and error functions.

Chan's method [5] is the only one based on an analytical formula rather than a numerical scheme. However, it is derived via an approximation of the domain of integration. Geometrically, Chan considers the integration problem of an isotropic Gaussian function over an ellipse - isotropic meaning that the level sets of the Gaussian function are circles. It is strictly equivalent to the original integration problem of an anisotropic Gaussian function over a disk. Then, the actual elliptic domain of integration is approximated by a disk of equivalent area and same center. Thus, the probability of collision reduces to the integral of an isotropic Gaussian function over a disk shifted from its peak. This integration problem can be solved analytically by use of properties of the first modified Bessel function. Finally, Chan is able to write the probability of collision as an infinite series with positive terms. The advantage of this approach is to provide the user with a truncation error that can be bounded. The major drawback lies in the fact that the formula itself is only an approximation of the original integral and that the discrepancy between the two is hard to quantify. One of the advantages of the present paper is to propose a different analytic formula for the probability of collision - derived without any approximation.

\section{A new method to compute the probability of collision}

The three main ingredients used in the derivation of the new formula are:

1. A method introduced by Lasserre and Zeron [11] to integrate Gaussian functions over Euclidean balls is used. Specifically, equation (4) is reformulated as a function $g(\xi)$, with $\xi=R^{2}$, whose Laplace transform $\mathcal{L}_{g}$ is computed in closed-form. The function is then expanded in a Laurent series. A term by term application of the inverse Laplace transform leads to a power series for the initial integral.

2. For the first time, a simple form for these coefficients is found. In Section III B, they are proved 
to satisfy a linear recurrence with polynomial coefficients (in the index variable). Classical theory and properties of D-finite (or holonomic) functions [12, 13] are used for this purpose.

3. From a numerical point of view, the direct evaluation of the series obtained for $g(\xi)$ can be difficult. Roughly speaking, although the power series expansion of $g(\xi)$ is convergent, the evaluation of the sum in finite precision arithmetic is prone to high cancellation $[17,18]$. This comes from consecutive terms that are close in magnitude, but of different signs, so that their sum in finite precision arithmetic contains very few correct significant digits. This makes the power series evaluation impractical for large values of $\xi$. Therefore, a so-called preconditionning $[17,18]$ is used in order to obtain a series suitable for low cancellation numerical evaluation. Instead of $g$, the function $\psi \cdot g$ is considered, where the so-called preconditionner $\psi$ is a D-finite function as well. By carefully choosing the preconditionner, both $\psi$ and $\psi \cdot g$ can be efficiently numerically evaluated.

In the end, the formula for the probability of collision has the following form:

$$
\mathcal{P}_{c}=\exp \left(-\frac{R^{2}}{2 \sigma_{y}^{2}}\right) \sum_{k=0}^{+\infty} \beta_{k} R^{2 k}
$$

where $\left(\beta_{k}\right)_{k \geq 0}$ is a positive sequence given by an explicit linear recurrence. This is detailed in what follows.

\section{A. Laplace transform computation of the preconditioned probability of collision}

The probability of collision given by (4) may be written as $\mathcal{P}_{c}=g\left(R^{2}\right)$ where the function $g: \mathbb{R}^{+} \mapsto \mathbb{R}^{+}$is defined as follows:

$$
g(\xi)=\frac{1}{2 \pi \sigma_{x} \sigma_{y}} \int_{\overline{\mathcal{B}}((0,0), \sqrt{\xi})} \exp \left(-\frac{1}{2}\left(\frac{\left(x-x_{m}\right)^{2}}{\sigma_{x}^{2}}+\frac{\left(y-y_{m}\right)^{2}}{\sigma_{y}^{2}}\right)\right) \mathrm{d} x \mathrm{~d} y
$$

This function is in fact the cumulative density function (cdf) of the random variable $\Xi=X^{2}+Y^{2}$ where $X \sim \mathcal{N}\left(x_{m}, \sigma_{x}^{2}\right)$ and $Y \sim \mathcal{N}\left(y_{m}, \sigma_{y}^{2}\right)$ are independent random variables.

The function $f=\psi \cdot g$ is considered instead of the function $g$ to prevent the cancellation phenomenon. The heuristic for a good choice of $\psi$ is based on a method presented in [18] (using several complex analysis results and properties obtained for $g$ ). In our case, it may be proved that 
an exponential function $\psi: \xi \mapsto \exp (p \xi)$ is a good choice for several values of $p$. The perconditioner $p=\frac{1}{2 \sigma_{y}^{2}}$ is chosen, since it leads to a simple proof of positivity of the series coefficients of $g$ and moreover, allows for a unified and generalized way of interpreting the exact analytic formula obtained by Chan [5, Chap. 4] in the isotropic case i.e., for $\sigma_{x}=\sigma_{y}$ (see Section IV). The following proposition provides the closed-form Laplace transform for $f$.

Proposition III.1. Let $f: \mathbb{R}^{+} \mapsto \mathbb{R}^{+}$, such that

$$
f(\xi)=\exp (p \xi) g(\xi)
$$

where $p \in \mathbb{R}^{+}$. Then, the Laplace transform $\mathcal{L}_{f}$ of the function $f$, is given by

$$
\mathcal{L}_{f}(\lambda)=\frac{\exp \left(-(\lambda-p)\left(\frac{x_{m}^{2}}{2(\lambda-p) \sigma_{x}^{2}+1}+\frac{y_{m}^{2}}{2(\lambda-p) \sigma_{y}^{2}+1}\right)\right)}{(\lambda-p) \sqrt{2(\lambda-p) \sigma_{x}^{2}+1} \sqrt{2(\lambda-p) \sigma_{y}^{2}+1}}, \text { for }|\lambda|>p .
$$

Proof. The function $g$ can be expressed as an integral over $\mathbb{R}^{2}$ by use of the set-indicator function:

$$
g(\xi)=\frac{1}{2 \pi \sigma_{x} \sigma_{y}} \int_{\mathbb{R}^{2}} \mathbf{1}_{\overline{\mathcal{B}}((0,0), \sqrt{\xi})}(x, y) \exp \left(-\frac{1}{2}\left(\frac{\left(x-x_{m}\right)^{2}}{\sigma_{x}^{2}}+\frac{\left(y-y_{m}\right)^{2}}{\sigma_{y}^{2}}\right)\right) \mathrm{d} x \mathrm{~d} y .
$$

Consider now the Laplace transform $\mathcal{L}_{g}$ of $g$. Then, for all $\lambda \in \mathbb{C}$ such that $\operatorname{Re}(\lambda)>0$,

$$
\begin{aligned}
\mathcal{L}_{g}(\lambda) & =\int_{0}^{+\infty} g(\xi) \exp (-\lambda \xi) \mathrm{d} \xi \\
& =\frac{1}{2 \pi \sigma_{x} \sigma_{y}} \int_{0}^{+\infty} \int_{\mathbb{R}^{2}} \mathbf{1}_{\overline{\mathcal{B}}((0,0), \sqrt{\xi})}(x, y) \exp \left(-\lambda \xi-\frac{1}{2}\left(\frac{\left(x-x_{m}\right)^{2}}{\sigma_{x}^{2}}+\frac{\left(y-y_{m}\right)^{2}}{\sigma_{y}^{2}}\right)\right) \mathrm{d} x \mathrm{~d} y \mathrm{~d} \xi
\end{aligned}
$$

Interchanging integrals is justified by Fubini's theorem, by absolute convergence of (13), that becomes:

$$
\begin{aligned}
\mathcal{L}_{g}(\lambda) & =\frac{1}{2 \pi \sigma_{x} \sigma_{y}} \int_{\mathbb{R}^{2}} \int_{0}^{+\infty} \mathbf{1}_{\overline{\mathcal{B}}((0,0), \sqrt{\xi})}(x, y) \exp (-\lambda \xi) \exp \left(-\frac{1}{2}\left(\frac{\left(x-x_{m}\right)^{2}}{\sigma_{x}^{2}}+\frac{\left(y-y_{m}\right)^{2}}{\sigma_{y}^{2}}\right)\right) \mathrm{d} \xi \mathrm{d} x \mathrm{~d} y \\
& =\frac{1}{2 \pi \sigma_{x} \sigma_{y} \lambda} \int_{\mathbb{R}^{2}} \exp \left(-\lambda\left(x^{2}+y^{2}\right)-\frac{1}{2}\left(\frac{\left(x-x_{m}\right)^{2}}{\sigma_{x}^{2}}+\frac{\left(y-y_{m}\right)^{2}}{\sigma_{y}^{2}}\right)\right) \mathrm{d} x \mathrm{~d} y \\
& =\frac{1}{2 \pi \sigma_{x} \sigma_{y} \lambda} \int_{-\infty}^{+\infty} \exp \left(-\lambda x^{2}-\frac{\left(x-x_{m}\right)^{2}}{2 \sigma_{x}^{2}}\right) \mathrm{d} x \int_{-\infty}^{+\infty} \exp \left(-\lambda y^{2}-\frac{\left(y-y_{m}\right)^{2}}{2 \sigma_{y}^{2}}\right) \mathrm{d} y .
\end{aligned}
$$

Next, each individual one-dimensional integral can be computed analytically. Indeed, after a change of variables aiming at completing the square and scaling the expression inside the exponential, the remaining integral is $\int_{-\infty}^{+\infty} \exp \left(-\frac{r^{2}}{2}\right) \mathrm{d} r=\sqrt{2 \pi}$. In the end, one gets:

$$
\mathcal{L}_{g}(\lambda)=\frac{\exp \left(-\lambda\left(\frac{x_{m}^{2}}{2 \lambda \sigma_{x}^{2}+1}+\frac{y_{m}^{2}}{2 \lambda \sigma_{y}^{2}+1}\right)\right)}{\lambda \sqrt{2 \lambda \sigma_{x}^{2}+1} \sqrt{2 \lambda \sigma_{y}^{2}+1}} .
$$


Multiplying the function $g(\xi)$ by $\exp (p \xi)$ corresponds to a translation in the Laplace domain $\mathcal{L}_{f}(\lambda)=\mathcal{L}_{g}(\lambda-p)$. Plugging this $p$-shift into (16) gives (11).

Note that in fact the function $\lambda \mapsto \lambda \mathcal{L}_{g}(-\lambda)$ is, by definition, the moment generating function of the random variable $\Xi$ previously defined [16].

\section{B. Power series of $f$ using D-finiteness}

For simplicity, the following notations are used: $p=\frac{1}{2 \sigma_{y}^{2}}, \phi=1-\frac{\sigma_{y}^{2}}{\sigma_{x}^{2}}, \omega_{x}=\frac{x_{m}^{2}}{4 \sigma_{x}^{4}}, \omega_{y}=\frac{y_{m}^{2}}{4 \sigma_{y}^{4}}$ and $\alpha_{0}=\frac{1}{2 \sigma_{x} \sigma_{y}} \exp \left(-\frac{1}{2}\left(\frac{x_{m}^{2}}{\sigma_{x}^{2}}+\frac{y_{m}^{2}}{\sigma_{y}^{2}}\right)\right)$.

Note that $0 \leq \phi<1, \omega_{x} \geq 0, \omega_{y} \geq 0$ and $\alpha_{0}>0$. With this notation, equation (11) becomes:

$$
\mathcal{L}_{f}(\lambda)=\frac{\alpha_{0} \exp \left(\frac{\omega_{y}}{\lambda}+\frac{\omega_{x}}{\lambda-p \phi}\right)}{\sqrt{\lambda(\lambda-p \phi)}(\lambda-p)}
$$

From (17), it is possible to obtain the power series of $f$ by performing a term-by-term inverse Laplace Transform on the principal part of Laurent series (i.e., the series of terms with negative degree) of $\mathcal{L}_{f}$ [19, Chap. 9], [20, Chap. 2.14].

Note first that the principal part of the Laurent series of $\mathcal{L}_{f}$ can be obtained by computing the Taylor expansion at infinity of $\mathcal{L}_{f}$. This is done by computing the coefficients of the power series expansion (at zero) of $\mathcal{L}_{f}\left(\lambda^{-1}\right)$. Since the first two coefficients of $\mathcal{L}_{f}\left(\lambda^{-1}\right)$ are zero, let $\hat{\mathcal{L}}_{f}(\lambda):=\lambda^{-2} \mathcal{L}_{f}\left(\lambda^{-1}\right)$ in order to simplify subsequent formulas. The multiplication by $\lambda^{-2}$ is a technicality: it means just a shift by two positions in the series coefficients.

A simple computation gives $\mathcal{L}_{f}\left(\lambda^{-1}\right)=\frac{\alpha_{0} \lambda^{2} \exp \left(\omega_{y} \lambda-\frac{\omega_{x}}{p \phi}-\frac{\omega_{x}}{p \phi(p \phi \lambda-1)}\right)}{\sqrt{1-p \phi \lambda}(1-p \lambda)}$. A simple derivation shows that $\hat{\mathcal{L}}_{f}$ verifies:

$$
\frac{\mathrm{d} \hat{\mathcal{L}}_{f}(\lambda)}{\mathrm{d} \lambda}=\varphi(\lambda) \hat{\mathcal{L}}_{f}(\lambda), \quad \hat{\mathcal{L}}_{f}(0)=\alpha_{0},
$$

where

$$
\varphi(\lambda)=\omega_{y}-\frac{p \phi}{2(p \phi \lambda-1)}-\frac{p}{p \lambda-1}+\frac{\omega_{x}}{(p \phi \lambda-1)^{2}}
$$

Hence, $\hat{\mathcal{L}}_{f}(\lambda)$ is D-finite and may be expanded in $\sum_{k=0}^{\infty} \alpha_{k} \lambda^{k}$, for all $\left\{\lambda,|\lambda|<\frac{1}{p}\right\}$. Therefore, the 
sequence $\left(\alpha_{k}\right)_{k \geq 0}$ satisfies a linear recurrence obtained using Gfun and given by:

$$
\begin{array}{r}
(k+4) \alpha_{k+4}=-p^{3} \phi^{2} \omega_{y} \alpha_{k}+p^{2} \phi\left(p \phi\left(k+\frac{5}{2}\right)+2 \omega_{y}\left(\frac{\phi}{2}+1\right)\right) \alpha_{k+1} \\
-p\left(p \phi\left(\frac{\phi}{2}+1\right)(2 k+5)+\phi\left(2 \omega_{y}+\frac{3 p}{2}\right)+\omega_{x}+\omega_{y}\right) \alpha_{k+2} \\
+\left(p(2 \phi+1)(k+3)+p\left(\frac{\phi}{2}+1\right)+\omega_{x}+\omega_{y}\right) \alpha_{k+3}
\end{array}
$$

with initial conditions: $\alpha_{0}$ given above,

$$
\begin{aligned}
\alpha_{1} & =\alpha_{0}\left(p\left(\frac{\phi}{2}+1\right)+\omega_{x}+\omega_{y}\right) \\
\alpha_{2} & =\frac{\alpha_{0}}{2}\left(\left(p\left(\frac{\phi}{2}+1\right)+\omega_{x}+\omega_{y}\right)^{2}+p^{2}\left(\frac{\phi^{2}}{2}+1\right)+2 p \phi \omega_{x}\right), \\
\alpha_{3} & =\frac{\alpha_{0}}{6}\left(\left(p\left(\frac{\phi}{2}+1\right)+\omega_{x}+\omega_{y}\right)^{3}+3\left(p\left(\frac{\phi}{2}+1\right)+\omega_{x}+\omega_{y}\right)\left(p^{2}\left(\frac{\phi^{2}}{2}+1\right)+2 p \phi \omega_{x}\right)\right. \\
& \left.+2\left(p^{3}\left(\frac{\phi^{3}}{2}+1\right)+3 p^{2} \phi^{2} \omega_{x}\right)\right) .
\end{aligned}
$$

The final formula for the convergent series expansion of $f$ and the positivity of the coefficients of this series is stated in the following theorem.

Theorem III.2. The convergent series expansion of $f$ is:

$$
f(\xi)=\sum_{k=0}^{+\infty} \beta_{k} \xi^{k}
$$

where

$$
\beta_{0}=0, \beta_{k+1}=\frac{\alpha_{k}}{(k+1) !}
$$

$\left(\alpha_{k}\right)_{k \geq 0}$ are defined in equations (20), (21) and $\beta_{k}>0$, for all $k \geq 0$.

Proof. The equation (23) can be seen as a term-by-term Inverse Laplace Transform, since $\mathcal{L}^{-1}\left(\frac{\alpha_{k}}{\lambda^{k+2}}\right)=\frac{\alpha_{k} \xi^{k+1}}{(k+1) !}$. The proper justification for this is based on [19, Chap. 9]. Moreover, in can be proven using complex analysis arguments [20, Chap. 2.14] that $f$ is an entire function, which implies the convergence of the series in equation (22). The positivity of the coefficients $\beta_{k}$ is deduced from the positivity of the coefficients $\alpha_{k}$. Indeed, since $\varphi(\lambda)=\sum_{k=0}^{\infty} \varphi_{k} \lambda^{k}$ is a rational fraction, the general closed-form for its power series coefficients $\varphi_{k}$ can be obtained by hand, based 
on the partial fraction decomposition of $\varphi$ (or using ratpolytocoeff Gfun procedure):

$$
\varphi_{k}=p^{k+1}\left(1+\phi^{k}\left(\frac{\omega_{x}(k+1)}{p}+\frac{\phi}{2}\right)\right)+ \begin{cases}0, & k>0 \\ \omega_{y}, & k=0 .\end{cases}
$$

It follows that $\varphi_{k}>0$, for all $k \in \mathbb{N}$. Injecting the series $\sum_{k=0}^{\infty} \varphi_{k} \lambda^{k}$ and $\sum_{k=0}^{\infty} \alpha_{k} \lambda^{k}$ into the differential equation (18) and extracting the coefficient of $\lambda^{k}$, one gets:

$$
(k+1) \alpha_{k+1}=\sum_{i=0}^{k} \varphi_{i} \alpha_{k-i},
$$

and hence, since $\alpha_{0}>0$, it follows that $\alpha_{k}>0$, for all $k \in \mathbb{N}$ and therefore $\beta_{k}>0$, for all $k \in \mathbb{N}$.

This entails the final analytic series form of the collision probability formula:

$$
\mathcal{P}_{c}=g\left(R^{2}\right)=\exp \left(-p R^{2}\right) \sum_{k=0}^{+\infty} \beta_{k} R^{2 k}
$$

For an efficient computation of truncations of this series, it is important to remark that also the terms $\beta_{k} R^{2 k}$ satisfy the following linear recurrence:

Proposition III.3. Let $c_{k}=\beta_{k} R^{2 k}$. Then

$$
\mathcal{P}_{c}=\exp \left(-p R^{2}\right) \sum_{k=0}^{+\infty} c_{k}
$$

and $\left(c_{k}\right)_{k \geq 0}$ satisfies:

$$
\begin{array}{r}
(k+4) c_{k+4}=-\frac{R^{8} p^{3} \phi^{2} \omega_{y}}{(k+2)(k+3)(k+4)(k+5)} c_{k}+\frac{R^{6} p^{2} \phi\left(p \phi\left(k+\frac{5}{2}\right)+2 \omega_{y}\left(\frac{\phi}{2}+1\right)\right)}{(k+3)(k+4)(k+5)} c_{k+1} \\
-\frac{R^{4} p\left(p \phi\left(\frac{\phi}{2}+1\right)(2 k+5)+\phi\left(2 \omega_{y}+\frac{3 p}{2}\right)+\omega_{x}+\omega_{y}\right)}{(k+4)(k+5)} c_{k+2} \\
+\frac{R^{2}\left(p(2 \phi+1)(k+3)+p\left(\frac{\phi}{2}+1\right)+\omega_{x}+\omega_{y}\right)}{k+5} c_{k+3},
\end{array}
$$

with initial conditions

$$
\begin{aligned}
c_{0} & =\alpha_{0} R^{2}, \\
c_{1} & =\frac{\alpha_{0} R^{4}}{2}\left(p\left(\frac{\phi}{2}+1\right)+\omega_{x}+\omega_{y}\right), \\
c_{2} & =\frac{\alpha_{0} R^{6}}{6}\left(\left(p\left(\frac{\phi}{2}+1\right)+\omega_{x}+\omega_{y}\right)^{2}+p^{2}\left(\frac{\phi^{2}}{2}+1\right)+2 p \phi \omega_{x}\right), \\
c_{3} & =\frac{\alpha_{0} R^{8}}{24}\left(\left(p\left(\frac{\phi}{2}+1\right)+\omega_{x}+\omega_{y}\right)^{3}+3\left(p\left(\frac{\phi}{2}+1\right)+\omega_{x}+\omega_{y}\right)\left(p^{2}\left(\frac{\phi^{2}}{2}+1\right)+2 p \phi \omega_{x}\right)\right. \\
& \left.+2\left(p^{3}\left(\frac{\phi^{3}}{2}+1\right)+3 p^{2} \phi^{2} \omega_{x}\right)\right) .
\end{aligned}
$$


Proof. The recurrence for $c_{k}$ is a direct computation (by hand or with Gfun), obtained from the recurrence on $\alpha_{k}$ (see equation (20)).

Truncating the series in (27) gives a straightforward algorithm (see Algorithm 1) for computing the probability of collision $\mathcal{P}_{c}$. Besides the fact that this series is the exact analytic formula for the probability, this algorithm features numerical stability. The coefficients $c_{k}$ are positive, so partial sums of the series already provide a lower bound for the exact value of $\mathcal{P}_{c}$. Note that this algorithm requires two exponential function evaluations and $O(N)$ basic arithmetic operations, where $N$ is the number of terms in the partial sum.

Algorithm 1 Computation of the Probability of Collision.

Input: Parameters: $\sigma_{x}, \sigma_{y}, x_{m}, y_{m}$; combined object radius: $R$; Number of terms: $N$.

Output: $\tilde{\mathcal{P}}_{c}-$ truncated series approximation of $\mathcal{P}_{c}$.

1: $p=\frac{1}{2 \sigma_{y}^{2}} ; \phi=1-\frac{\sigma_{y}^{2}}{\sigma_{x}^{2}} ; \omega_{x}=\frac{x_{m}^{2}}{4 \sigma_{x}^{4}} ; \omega_{y}=\frac{y_{m}^{2}}{4 \sigma_{y}^{4}} ; \alpha_{0}=\frac{1}{2 \sigma_{x} \sigma_{y}} \exp \left(-\frac{1}{2}\left(\frac{x_{m}^{2}}{\sigma_{x}^{2}}+\frac{y_{m}^{2}}{\sigma_{y}^{2}}\right)\right)$;

2: $c_{0}=\alpha_{0} R^{2}$

3: $c_{1}=\frac{\alpha_{0} R^{4}}{2}\left(p\left(\frac{\phi}{2}+1\right)+\omega_{x}+\omega_{y}\right)$;

4: $c_{2}=\frac{\alpha_{0} R^{6}}{6}\left(\left(p\left(\frac{\phi}{2}+1\right)+\omega_{x}+\omega_{y}\right)^{2}+p^{2}\left(\frac{\phi^{2}}{2}+1\right)+2 p \phi \omega_{x}\right)$;

5 :

$c_{3}=\frac{\alpha_{0} R^{8}}{24}\left(\left(p\left(\frac{\phi}{2}+1\right)+\omega_{x}+\omega_{y}\right)^{3}+3\left(p\left(\frac{\phi}{2}+1\right)+\omega_{x}+\omega_{y}\right)\left(p^{2}\left(\frac{\phi^{2}}{2}+1\right)+2 p \phi \omega_{x}\right)\right.$

$\left.+2\left(p^{3}\left(\frac{\phi^{3}}{2}+1\right)+3 p^{2} \phi^{2} \omega_{x}\right)\right)$;

6: for $k \leftarrow 0$ to $N-5$ do

$c_{k+4}=-\frac{R^{8} p^{3} \phi^{2} \omega_{y}}{(k+2)(k+3)(k+4)^{2}(k+5)} c_{k}+\frac{R^{6} p^{2} \phi\left(p \phi\left(k+\frac{5}{2}\right)+2 \omega_{y}\left(\frac{\phi}{2}+1\right)\right)}{(k+3)(k+4)^{2}(k+5)} c_{k+1}$

$7:$

$$
\begin{aligned}
& -\frac{R^{4} p\left(p \phi\left(\frac{\phi}{2}+1\right)(2 k+5)+\phi\left(2 \omega_{y}+\frac{3 p}{2}\right)+\omega_{x}+\omega_{y}\right)}{(k+4)^{2}(k+5)} c_{k+2} \\
& +\frac{R^{2}\left(p(2 \phi+1)(k+3)+p\left(\frac{\phi}{2}+1\right)+\omega_{x}+\omega_{y}\right)}{(k+4)(k+5)} c_{k+3}
\end{aligned}
$$

8: end for

9: $s \leftarrow 0$

10: for $k \leftarrow 0$ to $N-1$ do

11: $s \leftarrow s+c_{k} ;$

12: end for

13: $\tilde{\mathcal{P}}_{c} \leftarrow \exp \left(-p R^{2}\right) s$

14: return $\tilde{\mathcal{P}}_{c}$. 


\section{Computing the probability of collision with guaranteed accuracy}

While Algorithm 1 provides already a lower bound for $\mathcal{P}_{c}$, it is useful to also have an upper bound for the truncation error entailed by computing the partial sum $\tilde{\mathcal{P}}_{n}=\exp \left(-p R^{2}\right) \sum_{k=0}^{n-1} c_{k}$. The choice of the order $n$ of truncation is strongly dependent upon the trade-off between the numerical complexity involved in the computation and the precision of the obtained result. Thus, it is important to have sharp order-dependent error estimates that will help the user to manage this trade-off. Let us first define what may be interpreted as the truncation of order $n$ :

$$
\varepsilon_{n}=\mathcal{P}_{c}-\tilde{\mathcal{P}}_{n}\left(R^{2}\right)
$$

where $\tilde{\mathcal{P}}_{n}=\exp \left(-p R^{2}\right) \sum_{k=0}^{n-1} c_{k}=\exp \left(-p R^{2}\right) \sum_{k=0}^{n-1} \frac{\alpha_{k}}{(k+1) !}\left(R^{2}\right)^{k+1}$. The objective of the next proposition is to give accurate bounds on $\varepsilon_{n}$. As will be seen in the following, truncation error bounds also allow for an a priori computation of the truncation order, when a pre-specified precision on the collision probability is required. To avoid rather tedious developments, the proofs of the propositions presented in this Section are gathered in Appendix A.

Proposition III.4. The following bounds hold for the truncation error:

$$
l_{n} \leq \mathcal{P}_{c}-\tilde{\mathcal{P}}_{n}\left(R^{2}\right) \leq u_{n}
$$

where

$$
l_{n}:=\frac{\alpha_{0} \exp \left(-p R^{2}\right)\left(p R^{2}\right)^{n+1}}{p(n+1) !}
$$

and

$$
u_{n}:=\frac{\alpha_{0} \exp \left(p\left(\frac{\phi}{2}+\frac{\omega_{x}+\omega_{y}}{p}\right) R^{2}\right)\left(p\left(1+\frac{\phi}{2}+\frac{\omega_{x}+\omega_{y}}{p}\right) R^{2}\right)^{n+1}}{p\left(1+\frac{\phi}{2}+\frac{\omega_{x}+\omega_{y}}{p}\right)(n+1) !},
$$

for all $n \geq 1$.

These bounds are used for computing the probability of collision with a guaranteed accuracy. Let $\delta$ be the probability threshold for executing some collision avoidance maneuver, say $\delta=0.001$ in practice. The test $\mathcal{P}_{c} \leq \delta$ needs to be performed safely, which means that at least $\left\lceil-\log _{10} \delta\right\rceil$ digits need to be guaranteed in the computation of $\mathcal{P}_{c}$. Practically, for $\delta=0.001,3$ or 4 guaranteed digits (after the decimal dot, that is in absolute error) should be sufficient. In the following proposition, a sufficient value for $n$ is given such that the required accuracy holds. 
Proposition III.5. Let

$$
\begin{gathered}
N_{1}=2\left\lceil e p R^{2}\left(1+\frac{\phi}{2}+\frac{\omega_{x}+\omega_{y}}{p}\right)\right\rceil, \\
N_{2}=\left\lceil\log _{2} \frac{\alpha_{0} \exp \left(p R^{2}\left(\frac{\phi}{2}+\frac{\omega_{x}+\omega_{y}}{p}\right)\right)}{\delta p \sqrt{2 \pi N_{1}}\left(1+\frac{\phi}{2}+\frac{\omega_{x}+\omega_{y}}{p}\right)}\right\rceil,
\end{gathered}
$$

and

$$
n+1=\max \left\{N_{1}, N_{2}\right\}
$$

Then, $u_{n}-l_{n}<\delta$.

In the proof of Proposition III.4 presented in Appendix A, Proposition A.3 provides two majorant/minorant series $\bar{\varepsilon}_{n}$ and $\underline{\varepsilon}_{n}$ for the truncation error $\varepsilon_{n}$. These can be also used to obtain directly an upper/lower bound for the probability $\mathcal{P}_{c}$ as stated below.

Proposition III.6. Let

$$
l_{0}=\alpha_{0} \frac{1-\exp \left(-p R^{2}\right)}{p}
$$

and

$$
u_{0}=\alpha_{0} \frac{\exp \left(p\left(\frac{\phi}{2}+\frac{\omega_{x}+\omega_{y}}{p}\right) R^{2}\right)-\exp \left(-p R^{2}\right)}{p\left(1+\frac{\phi}{2}+\frac{\omega_{x}+\omega_{y}}{p}\right)} .
$$

Then:

$$
l_{0} \leq \mathcal{P}_{c} \leq u_{0}
$$

The formulas obtained for $l_{0}$ and $u_{0}$ are simple and sufficient in most practical cases. They provide a guaranteed enclosure for the actual value of the probability (see Section V, Table 2 for numerical examples).

Propositions III.6, III.4 and III.5 allow for computing an enclosure of the exact value of the probability with a guaranteed absolute error less then a given threshold $\delta$. This is implemented in Algorithm 2. The advantage of this algorithm is to allow for an a priori computation of a sufficient number $n$ of terms to be taken in the series such that the accuracy requirement holds. This algorithm 
calls Algorithm 1 (line 10, Algorithm 2) to obtain the sum of the truncated series of $n$ terms i.e., an approximation $\tilde{\mathcal{P}}_{c}$ of the probability of collision. The variables $l_{n}$ and $u_{n}$ (line 11,12 , Algorithm 2 ) provide lower and upper bounds on the truncation error. Whence, one obtains an effective enclosure of the true value of the probability in lines 13, 14 of Algorithm 2. Numerical results are given in Section V (see Figure 3 in particular).

Algorithm 2 Computation of probability of collision with guaranteed accuracy.

Input: Parameters: $\sigma_{x}, \sigma_{y}, x_{m}, y_{m}$; combined object radius: $R$; Threshold $\delta$.

Output: $\left[\underline{\mathcal{P}}_{c}, \overline{\mathcal{P}}_{c}\right]$ such that $\underline{\mathcal{P}}_{c} \leq \mathcal{P}_{c} \leq \overline{\mathcal{P}}_{c}$ and $\overline{\mathcal{P}}_{c}-\underline{\mathcal{P}}_{c} \leq \delta$.

$1: p=\frac{1}{2 \sigma_{y}^{2}} ; \phi=1-\frac{\sigma_{y}^{2}}{\sigma_{x}^{2}} ; \omega_{x}=\frac{x_{m}^{2}}{4 \sigma_{x}^{4}} ; \omega_{y}=\frac{y_{m}^{2}}{4 \sigma_{y}^{4}} ; \alpha_{0}=\frac{1}{2 \sigma_{x} \sigma_{y}} \exp \left(-\frac{1}{2}\left(\frac{x_{m}^{2}}{\sigma_{x}^{2}}+\frac{y_{m}^{2}}{\sigma_{y}^{2}}\right)\right)$;

2: $l_{0}=\alpha_{0} \frac{1-\exp \left(-p R^{2}\right)}{p}$;

3: $u_{0}=\alpha_{0} \frac{\exp \left(p\left(\frac{\phi}{2}+\frac{\omega_{x}+\omega_{y}}{p}\right) R^{2}\right)-\exp \left(-p R^{2}\right)}{p\left(1+\frac{\phi}{2}+\frac{\omega_{x}+\omega_{y}}{p}\right)} ;$

4: if $u_{0}-l_{0} \leq \delta$ then

5: return $\left[l_{0}, u_{0}\right]$;

6: else

7: $N_{1}=2\left\lceil e p R^{2}\left(1+\frac{\phi}{2}+\frac{\omega_{x}+\omega_{y}}{p}\right)\right\rceil ;$

8: $N_{2}=\left\lceil\log _{2} \frac{\alpha_{0} \exp \left(p R^{2}\left(\frac{\phi}{2}+\frac{\omega_{x}+\omega_{y}}{p}\right)\right)}{\delta p \sqrt{2 \pi N_{1}}\left(1+\frac{\phi}{2}+\frac{\omega_{x}+\omega_{y}}{p}\right)}\right\rceil$;

9: $n=\max \left\{N_{1}, N_{2}\right\}-1$

10: $\tilde{\mathcal{P}}_{c} \leftarrow$ Algorithm $1\left(\sigma_{x}, \sigma_{y}, x_{m}, y_{m}, R, n\right)$;

11: $l_{n}=\frac{\alpha_{0} \exp \left(-p R^{2}\right)\left(p R^{2}\right)^{n+1}}{p(n+1) !}$;

12: $u_{n}=\frac{\alpha_{0} \exp \left(p\left(\frac{\phi}{2}+\frac{\omega_{x}+\omega_{y}}{p}\right) R^{2}\right)\left(p\left(1+\frac{\phi}{2}+\frac{\omega_{x}+\omega_{y}}{p}\right) R^{2}\right)^{n+1}}{p\left(1+\frac{\phi}{2}+\frac{\omega_{x}+\omega_{y}}{p}\right)(n+1) !}$;

13: $\underline{\mathcal{P}}_{c}=\tilde{\mathcal{P}}_{c}+l_{n}$

14: $\overline{\mathcal{P}}_{c}=\tilde{\mathcal{P}}_{c}+u_{n}$

15: return $\left[\underline{\mathcal{P}}_{c}, \overline{\mathcal{P}}_{c}\right]$.

16: end if

\section{Comparison with Chan series}

In the isotropic case (i.e., $\sigma_{x}=\sigma_{y}:=\sigma$ ), Chan [5, Chap. 4] reformulates the initial 2-D integral (4) as a one dimension integral over the Rice probability distribution. This integral is then computed analytically in the form of a power series using some knowledge (identities, integral 
tables) on the modified Bessel function of the first kind and the Rice distribution. We recover the same series applying our procedure described in Section III B. In the isotropic case, the Laplace transform in equation (16) is:

$$
\mathcal{L}_{g_{\text {iso }}}(\lambda)=\frac{\exp \left(\frac{x_{m}^{2}+y_{m}^{2}}{\sigma^{2}\left(2 \sigma^{2} \lambda+1\right)}\right)}{\lambda\left(2 \sigma^{2} \lambda+1\right)} .
$$

Applying a similar preconditionning method [17, 18], a preconditioning parameter $p=\frac{1}{2 \sigma^{2}}$ is derived. A result similar to Theorem III.2 is easily derived. Note that since $\sigma_{x}=\sigma_{y}$, one has $\phi=0$, $\omega_{x}+\omega_{y}=\frac{x_{m}^{2}+y_{m}^{2}}{4 \sigma^{4}}$ and $\alpha_{0}=\frac{1}{2 \sigma^{2}} \exp \left(-\frac{x_{m}^{2}+y_{m}^{2}}{2 \sigma^{2}}\right)$. Define $\hat{\mathcal{L}}_{f_{\text {iso }}}(\lambda):=\lambda^{-2} \mathcal{L}_{f_{\text {iso }}}\left(\lambda^{-1}\right)$, then

$$
\frac{\mathrm{d} \hat{\mathcal{L}}_{f_{\text {iso }}}(\lambda)}{\mathrm{d} \lambda}=\varphi_{\text {iso }}(\lambda) \hat{\mathcal{L}}_{f_{\text {iso }}}(\lambda), \quad \hat{\mathcal{L}}_{f_{\text {iso }}}(0)=\alpha_{0},
$$

where

$$
\varphi_{\text {iso }}(\lambda)=\omega_{x}+\omega_{y}-\frac{p}{p \lambda-1}
$$

$\hat{\mathcal{L}}_{f_{\text {iso }}}(\lambda)$ can be expanded in $\sum_{k=0}^{+\infty} \alpha_{k} \lambda^{k}$, for all $\{\lambda,|\lambda|>p\}$ and $\left(\alpha_{k}\right)_{k \geq 0}$ satisfies

$$
(k+2) \alpha_{k+2}=-p\left(\omega_{x}+\omega_{y}\right) \alpha_{k}+\left(p(k+2)+\omega_{x}+\omega_{y}\right) \alpha_{k+1}
$$

with initial conditions: $\alpha_{0}$ given above and $\alpha_{1}=\alpha_{0}\left(p+\omega_{x}+\omega_{y}\right)$.

Proposition IV.1. The convergent series expansion of the function $f_{i s o}$ is:

$$
f_{i s o}(z)=\sum_{k=0}^{+\infty} \beta_{k} z^{k}
$$

where

$$
\beta_{0}=0, \beta_{k+1}=\frac{\alpha_{k}}{(k+1) !} \text { for all } k \geq 0,
$$

$\left(\alpha_{k}\right)_{k \geq 0}$ are defined in equation (40) and $\beta_{k}>0$, for all $k \geq 0$.

The advantage in this simpler case is that in (40), one has:

$$
(k+2)\left(\alpha_{k+2}-p \alpha_{k+1}\right)=\left(\omega_{x}+\omega_{y}\right)\left(\alpha_{k+1}-p \alpha_{k}\right),
$$

which easily gives the closed-form for $\alpha_{k+1}-p \alpha_{k}=\frac{\alpha_{0}\left(\omega_{x}+\omega_{y}\right)^{k+1}}{(k+1) !}$, and hence,

$$
\alpha_{k}=\alpha_{0} p^{k} \sum_{i=0}^{k} \frac{\left(\omega_{x}+\omega_{y}\right)^{i}}{p^{i} i !} .
$$


It follows that the final probability becomes:

$$
\begin{aligned}
\exp \left(-p R^{2}\right) \mathcal{P}_{\text {iso }}\left(R^{2}\right) & =\sum_{k=0}^{\infty} \frac{\alpha_{k} R^{2 k+2}}{(k+1) !} \\
& =\exp \left(-\frac{\omega_{x}+\omega_{y}}{p}\right) \sum_{k=0}^{\infty} \frac{\left(p R^{2}\right)^{k+1}}{(k+1) !} \sum_{i=0}^{k} \frac{\left(\omega_{x}+\omega_{y}\right)^{i}}{p^{i} i !} \\
& =\exp \left(-\frac{\omega_{x}+\omega_{y}}{p}\right) \sum_{i=0}^{\infty} \frac{\left(\omega_{x}+\omega_{y}\right)^{i}}{p^{i} i !} \sum_{k=i}^{\infty} \frac{\left(p R^{2}\right)^{k+1}}{(k+1) !}
\end{aligned}
$$

or equivalently,

$$
\mathcal{P}_{i s o}\left(R^{2}\right)=\exp \left(-\frac{\omega_{x}+\omega_{y}}{p}\right) \sum_{i=0}^{\infty} \frac{\left(\omega_{x}+\omega_{y}\right)^{i}}{p^{i} i !}\left(1-\exp \left(-p R^{2}\right) \sum_{k=0}^{i} \frac{\left(p R^{2}\right)^{k}}{k !}\right) .
$$

Formula (45) and equivalent ones obtained by simple re-summation are the ones used by Chan, see e.g., [5, eq. 4.17, Chap. 4], where the notation is $u=\frac{R^{2}}{\sigma^{2}}$ and $v=\frac{x_{m}^{2}+y_{m}^{2}}{\sigma^{2}}$.

In the non-isotropic case, Chan builds an approximation of the integral which allows for the following two changes of variables: $u=\frac{r^{2}}{\sigma_{x}^{2} \sigma_{y}^{2}}$ and $v=\frac{x_{m}^{2}}{\sigma_{y}^{2}}+\frac{y_{m}^{2}}{\sigma_{y}^{2}}$ in (45), used to compute the final approximated probability. In Chan's approach, a closed formula, namely equation (44), is maintained for the sequence $\alpha_{k}$ in the final formula for the non-isotropic case which remains an approximation of the genuine probability of collision. In contrast, the analytic formula obtained in this paper is exact and the sequence $\alpha_{k}$ (eq. (20)) is given by a linear recurrence which is numerically stable for a machine computation.

\section{Numerical tests}

The performance of our method is assessed from two perspectives. First, since it is based on a series expansion, the numerical accuracy varies in function of the number of terms computed. Algorithm 2 offers an automatic way of computing the number of terms needed for a user-required accuracy. We exemplify it in what follows on practical cases. Second, our method is compared with other methods from the literature concerning the quality of the results obtained. Three algorithms from the literature, namely Alfano's [4], Patera's [3] and respectively Chan's [5] have been implemented. The chosen test cases are described in Table 1: the first 12 cases can be found in [5, Chapter 5] and are supposed to be representative of real short-term encounters; the next 3 cases are real-case scenarios: the data were retrieved from CSMs (Conjunction Summary Messages) sent by 
the Joint Space Operations Center to the industrial partner of this study; the last two cases were obtained using the physical parameters of test cases number 3 and 5 provided by Alfano [7].

\begin{tabular}{|c|c|c|c|c|c|}
\hline \multirow{2}{*}{$\begin{array}{c}\text { Case } \\
\#\end{array}$} & \multicolumn{5}{|c|}{ Input parameters $(\mathrm{m})$} \\
\hline & $\sigma_{x}$ & $\sigma_{y}$ & $R$ & $x_{m}$ & $y_{m}$ \\
\hline Chan 1 & 50 & 25 & 5 & 10 & 0 \\
\hline Chan 2 & 50 & 25 & 5 & 0 & 10 \\
\hline Chan 3 & 75 & 25 & 5 & 10 & 0 \\
\hline Chan 4 & 75 & 25 & 5 & 0 & 10 \\
\hline Chan 5 & 3,000 & 1,000 & 10 & 1,000 & 0 \\
\hline Chan 6 & 3,000 & 1,000 & 10 & 0 & 1,000 \\
\hline Chan 7 & 3,000 & 1,000 & 10 & 10,000 & 0 \\
\hline Chan 8 & 3,000 & 1,000 & 10 & 0 & 10,000 \\
\hline Chan 9 & 10,000 & 1,000 & 10 & 10,000 & 0 \\
\hline Chan 10 & 10,000 & 1,000 & 10 & 0 & 10,000 \\
\hline Chan 11 & 3,000 & 1,000 & 50 & 5,000 & 0 \\
\hline Chan 12 & 3,000 & 1,000 & 50 & 0 & 5,000 \\
\hline CSM 1 & 152.8814468961533 & 57.918666623295984 & 10.3 & 60.583685340533115 & 84.875546447209487 \\
\hline CSM 1 & $5,756.840725983703$ & 15.988242371297744 & 1.3 & 115.0558998093139 & -81.618369910317043 \\
\hline CSM 3 & 643.4092722122279 & 94.230921098486149 & 5.3 & 693.4058939950484 & 102.1772470067133 \\
\hline Alfano 3 & 114.2585190378857 & 1.410183033040157 & 15 & 0.159164620813659 & -3.887207383647396 \\
\hline Alfano 5 & 177.8109003935867 & 0.037327944173609 & 10 & 2.123006718041866 & -1.221789517557463 \\
\hline
\end{tabular}

Table 1: Inputs for test cases from Chan (1-12), CSMs (1-3) and Alfano $(3,5)$

\section{A. Analysis of our method}

In Proposition III.6 two very simple formulas (evaluations of exponentials) are provided for guaranteed lower and upper bounds of the probability of collision. These two simple formulas (line 2, 3, Algorithm 2) provide enough accuracy in most practical cases, as shown in Table 2. Except in Alfano's cases, they seem to be very effective due to their simplicity and may suffice in most practical cases. 


\begin{tabular}{|c|c|c|c|}
\hline Case & Guaranteed Value & Relative Significant Digits & Absolute Significant Digits \\
\hline Chan 1 & $0.0097[04,75]$ & 2 & 4 \\
\hline Chan 2 & $0.0091[39,82]$ & 2 & 4 \\
\hline Chan 3 & $0.0065[42,72]$ & 2 & 4 \\
\hline Chan 4 & $0.006[09,13]$ & 1 & 3 \\
\hline Chan 5 & $0.15765[61,76] \cdot 10^{-4}$ & 5 & 9 \\
\hline Chan 6 & $0.10108[60,81] \cdot 10^{-4}$ & 5 & 9 \\
\hline Chan 7 & $0.6443[04,23] \cdot 10^{-7}$ & 4 & 11 \\
\hline Chan 8 & $0.321[45,86] \cdot 10^{-26}$ & 3 & 29 \\
\hline Chan 9 & $0.3032[58,61] \cdot 10^{-5}$ & 4 & 9 \\
\hline Chan 10 & $0.96[43,56] \cdot 10^{-27}$ & 2 & 29 \\
\hline Chan 11 & $0.1038[31,71] \cdot 10^{-3}$ & 4 & 7 \\
\hline Chan 12 & $0.15[18,65] \cdot 10^{-8}$ & 2 & 10 \\
\hline CSM 1 & $0.001[878,900]$ & 1 & 3 \\
\hline CSM 2 & $2.0[101,557] \cdot 10^{-11}$ & 2 & 13 \\
\hline CSM 3 & 7.[194, 200] $10^{-5}$ & 1 & 6 \\
\hline Alfano 3 & {$\left[0.27 \cdot 10^{-3}, 1.14 \cdot 10^{101}\right]$} & 0 & 0 \\
\hline Alfano 5 & {$\left[3.2 \cdot 10^{-228}, 3.8 \cdot 10^{7757429}\right]$} & 0 & 0 \\
\hline
\end{tabular}

Table 2: Guaranteed correct digits obtained with formulas in Proposition III.6. The notation e.g. $0.15[18,65] \cdot 10^{-8}$ stands for the fact that the probability is surely enclosed in the interval $\left[0.1518 \cdot 10^{-5}, 0.1565 \cdot 10^{-8}\right]$ and helps visualizing the guaranteed digits.

If more accuracy is needed, the subsequent lines of Algorithm 2 are employed. Figure 3 shows the number $n$ of series terms needed in Algorithm 2 for a requested accuracy $\delta$ ranging from $10^{-1}$ to $10^{-13}$. We observe that the number of terms for the absolute error to reach the machine precision $\left(10^{-13}\right)$ is less 40 in all of Chan's Cases and CSM cases. Note that this number is computed a priori and it is a sufficient number. The actual number of terms for the accuracy to be met may be smaller in some cases, but for these practical examples it is not conservative. Chan's cases 8 and 10 are not drawn since zero terms (that is the values computed in Table 2 with the simple bounds) 
are sufficient for the whole range of absolute error considered.

On the other hand, for Alfano's cases, our Algorithm needs much more terms. One observes in Table 2 that the bounds obtained for these two examples are meaningless. In order to reach a good accuracy, Algorithm 2 computes $n=689$ for Alfano's Case 3 and $n=10^{13}$ for Alfano's Case 5 . This is conservative, since 90 terms for Case 3 and respectively 37000 terms for Case 5 are sufficient to obtain the value given in Table 5. This shows however that in some degenerate cases the number of terms needed may increase drastically.
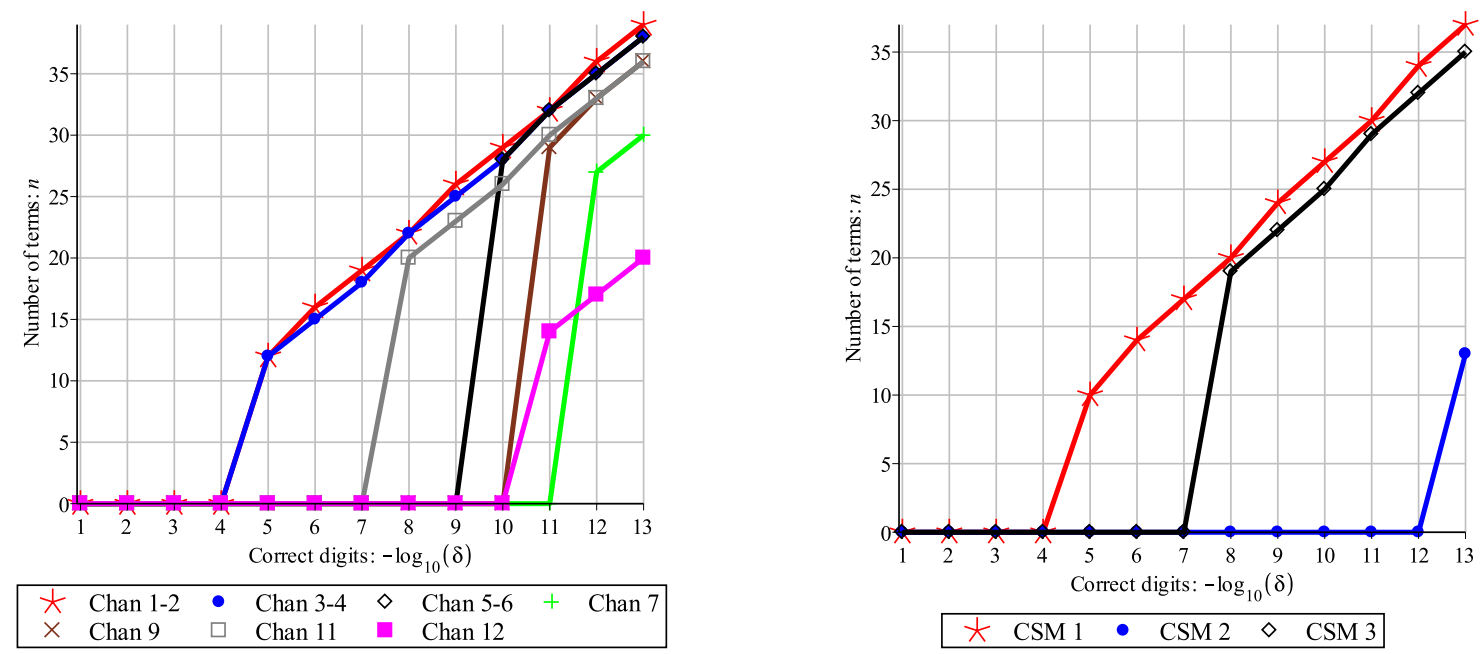

Figure 3: Number of terms in the series needed in Algorithm 2 for a requested accuracy $\delta$ ranging from $10^{-1}$ to $10^{-13}$. Cases shown: Chan's Cases (left) and CSM cases (right) from Table 1.

\section{B. Comparison with other methods}

The corresponding results for the probability of collision obtained with different methods are summarized in Tables 3, 4 and 5. The reference values in Table 3 were provided by NASA [5] using Foster's method. For Table 4, they were given by the industrial partner and for Table 5 they were obtained from Monte Carlo trials. All tests were performed with Matlab@ R2014a on an Intel@ $\mathrm{Xeon}(\mathrm{R})$ at $3.60 \mathrm{GHz}$.

Since in our method the required accuracy can be set a priori in Algorithm 2, the obtained values are identical (in most cases) or very close to the reference. For Chan's test cases, number 1 to 12 , Patera's method gives also $0 \%$ of relative error. For the same examples, Alfano's method 
also performs well, but it fails for very low probabilities like in test cases 8 and 10 . On the other hand, Chan's method gives non negligible relative errors for some cases - namely 1, 2, 3, 4, 8, 10, 12 and CSM1-3. As far as precision is concerned, it is definitely the least effective. It is not surprising since it is based on an additional approximation with respect to the original short-term encounter model. For that reason, it gives meaningless results for the two test cases provided by Alfano [7], see Table 5. These examples were originally designed to compare the efficiency of several methods of the literature and are somehow more tedious as far as computation is concerned. They are challenging also for our method, since the number of terms to be considered in the series expansion is important. Nevertheless, the new method gives satisfactory results.

Concerning timings, our method is very fast: for each case tested, the results are obtained in less than one second; in frequent cases, when the bounds $l_{0}$ and $u_{0}$ are sufficient (lines $2-3$, Algorithm 2), the response is almost instantaneous ( $10^{-5}$ seconds).

\begin{tabular}{|c|c|c|c|c|c|}
\hline \multirow{2}{*}{ Case } & \multicolumn{5}{|c|}{ Collision Probability $(-)$} \\
\cline { 2 - 6 } & Alfano & Patera & Chan & Algorithm 2 & Reference \\
\hline Chan 1 & $9.742 \times 10^{-3}$ & $9.741 \times 10^{-3}$ & $9.754 \times 10^{-3}$ & $9.742 \times 10^{-3}$ & $9.742 \times 10^{-3}$ \\
\hline Chan 2 & $9.181 \times 10^{-3}$ & $9.181 \times 10^{-3}$ & $9.189 \times 10^{-3}$ & $9.181 \times 10^{-3}$ & $9.181 \times 10^{-3}$ \\
\hline Chan 3 & $6.571 \times 10^{-3}$ & $6.571 \times 10^{-3}$ & $6.586 \times 10^{-3}$ & $6.571 \times 10^{-3}$ & $6.571 \times 10^{-3}$ \\
\hline Chan 4 & $6.125 \times 10^{-3}$ & $6.125 \times 10^{-3}$ & $6.135 \times 10^{-3}$ & $6.125 \times 10^{-3}$ & $6.125 \times 10^{-3}$ \\
\hline Chan 5 & $1.577 \times 10^{-5}$ & $1.577 \times 10^{-5}$ & $1.577 \times 10^{-5}$ & $1.577 \times 10^{-5}$ & $1.577 \times 10^{-5}$ \\
\hline Chan 6 & $1.011 \times 10^{-5}$ & $1.011 \times 10^{-5}$ & $1.011 \times 10^{-5}$ & $1.011 \times 10^{-5}$ & $1.011 \times 10^{-5}$ \\
\hline Chan 7 & $6.443 \times 10^{-8}$ & $6.443 \times 10^{-8}$ & $6.443 \times 10^{-8}$ & $6.443 \times 10^{-8}$ & $6.443 \times 10^{-8}$ \\
\hline Chan 8 & $\mathbf{0}$ & $3.219 \times 10^{-27}$ & $3.216 \times 10^{-27}$ & $3.219 \times 10^{-27}$ & $3.219 \times 10^{-27}$ \\
\hline Chan 9 & $3.033 \times 10^{-6}$ & $3.033 \times 10^{-6}$ & $3.033 \times 10^{-6}$ & $3.033 \times 10^{-6}$ & $3.033 \times 10^{-6}$ \\
\hline Chan 10 & $\mathbf{0}$ & $9.656 \times 10^{-28}$ & $9.645 \times 10^{-28}$ & $9.656 \times 10^{-28}$ & $9.656 \times 10^{-28}$ \\
\hline Chan 11 & $1.039 \times 10^{-4}$ & $1.039 \times 10^{-4}$ & $1.039 \times 10^{-4}$ & $1.039 \times 10^{-4}$ & $1.039 \times 10^{-4}$ \\
\hline Chan 12 & $1.564 \times 10^{-9}$ & $1.564 \times 10^{-9}$ & $1.556 \times 10^{-9}$ & $1.564 \times 10^{-9}$ & $1.564 \times 10^{-9}$ \\
\hline
\end{tabular}

Table 3: Comparison of collision probability value -with 4 significant digits- for Chan's test cases number 1 to 12 . The digits different from the reference value are represented in bold. 


\begin{tabular}{|c|c|c|c|c|c|}
\hline \multirow{2}{*}{ Case } & \multicolumn{5}{|c|}{ Collision Probability (-) } \\
\cline { 2 - 6 } & Alfano & Patera & Chan & Algorithm 2 & Reference \\
\hline CSM 1 & $1.9002 \times 10^{-3}$ & $1.9001 \times 10^{-3}$ & $1.8934 \times 10^{-3}$ & $1.9002 \times 10^{-3}$ & $1.9002 \times 10^{-3}$ \\
\hline CSM 2 & $2.0553 \times 10^{-11}$ & $2.0552 \times 10^{-11}$ & $2.0135 \times 10^{-11}$ & $2.0553 \times 10^{-11}$ & $2.0553 \times 10^{-11}$ \\
\hline CSM 3 & $7.2004 \times 10^{-5}$ & $7.2000 \times 10^{-5}$ & $7.2000 \times 10^{-5}$ & $7.2003 \times 10^{-5}$ & $7.2003 \times 10^{-5}$ \\
\hline
\end{tabular}

Table 4: Comparison of collision probability value -with 5 significant digits- for tests cases from CSMs. The digits different from the reference value are represented in bold.

\begin{tabular}{|c|c|c|c|c|c|}
\hline \multirow{2}{*}{ Case } & \multicolumn{5}{|c|}{ Collision Probability (-) } \\
\cline { 2 - 6 } & Alfano & Patera & Chan & Algorithm 2 & Reference \\
\hline Alfano's No. 3 & $1.0038 \times 10^{-1}$ & $1.0087 \times 10^{-1}$ & $3.1264 \times 10^{-2}$ & $1.0038 \times 10^{-1}$ & $1.0085 \times 10^{-1}$ \\
\hline Alfano's No. 5 & $4.4712 \times 10^{-2}$ & $4.4520 \times 10^{-2}$ & $1.6618 \times 10^{-77}$ & $4.4509 \times 10^{-2}$ & $4.4499 \times 10^{-2}$ \\
\hline
\end{tabular}

Table 5: Comparison of collision probability value - with 5 significant digits - for test cases from [7].

\section{Conclusions}

A new method to compute the probability of collision for short-term space encounters between two spherical objects under Gaussian-distributed uncertainty has been proposed. The formula has the form of a product between an exponential term and a convergent power series with positive coefficients. It is derived by use of Laplace transform theory and D-finite functions properties. The variable in the power series is the squared radius of the combined object. The series coefficients depend on the other parameters of the encounter, namely the miss distance and the standard deviations of the relative position of the objects in the encounter plane. It was shown that the formula is in fact an improvement on the only other analytical method of the literature - namely Chan's - since it is more general. Compared with state-of-the-art numerical methods, this work has several advantages. First of all, it provides an accurate algorithm to compute the probability of collision based on an analytic expression. Moreover, it gives a fast way to compute an estimate of the risk, which is often close-enough to the real value for the decision maker.

As a short term objective, we intend to address, in a finer manner, the cases for which the 
number of terms to be considered in the series expansion is too high. We will use for that purpose, a saddle-point based method [21, Chapter VIII]. In addition, a parameter sensitivity analysis similar to the one done by Chan, would be useful since we expect to obtain similar results (although with a better accuracy on the probability, due to the advantages of our method).

\section{Appendix A: DERIVATION OF THE BOUNDS ON THE TRUNCATION ERROR}

\section{Proof of Proposition III.4}

In order to prove the Proposition III.4, some preliminary results are needed. First, remember that

$$
\varepsilon_{n}=\mathcal{P}_{c}-\tilde{\mathcal{P}}_{n}\left(R^{2}\right)=\mathcal{P}_{c}-\exp \left(-p R^{2}\right) \sum_{k=0}^{n-1} \beta_{k}\left(R^{2}\right)^{k+1}=\mathcal{P}_{c}-\exp \left(-p R^{2}\right) \sum_{k=0}^{n-1} \frac{\alpha_{k}}{(k+1) !}\left(R^{2}\right)^{k+1}
$$

Then, recall that $\left(\alpha_{k}\right)_{k \geq 0}$ is known only through its recurrence and a closed formula is unlikely to be found. So, in order to compute effective truncation error bounds, two sequences $\left(\bar{\alpha}_{k}\right)_{k \geq 0}$ and $\left(\underline{\alpha}_{k}\right)_{k \geq 0}$ are used, which have closed-forms and provide respectively upper and lower bounds for $\left(\alpha_{k}\right)_{k \geq 0}$.

Proposition A.1. Let $\underline{\alpha}_{k}=\alpha_{0} p^{k}$ and $\bar{\alpha}_{k}=\alpha_{0} p^{k}\left(1+\frac{\phi}{2}+\frac{\omega_{x}+\omega_{y}}{p}\right)^{k}$. Then $\underline{\alpha}_{k} \leq \alpha_{k} \leq \bar{\alpha}_{k}$, for all $k \in \mathbb{N}$.

Proof. The proof follows by induction. First, one has $\alpha_{1}=\alpha_{0} \varphi_{0}=\alpha_{0} p\left(1+\frac{\phi}{2}+\frac{\omega_{x}+\omega_{y}}{p}\right)$, so

$$
\underline{\alpha}_{1}=\alpha_{0} p \leq \alpha_{0} \varphi_{0} \leq \alpha_{0} p\left(1+\frac{\phi}{2}+\frac{\omega_{x}+\omega_{y}}{p}\right)=\bar{\alpha}_{1} .
$$

To prove the induction step, let us first note:

$$
\frac{\alpha_{0} \varphi_{k}}{\underline{\alpha}_{k+1}}=1+\phi^{k}\left(\frac{\omega_{x}(k+1)}{p}+\frac{\phi}{2}\right)+ \begin{cases}0, & k>0 \\ \frac{\omega_{y}}{p}, & k=0 .\end{cases}
$$

and

$$
\frac{\alpha_{0} \varphi_{k}}{\bar{\alpha}_{k+1}}= \begin{cases}\frac{1+\phi^{k}\left(\frac{\omega_{x}(k+1)}{p}+\frac{\phi}{2}\right)}{\left(1+\frac{\phi}{2}+\frac{\omega_{x}+\omega_{y}}{p}\right)^{k+1}}, & k>0 \\ 1, & k=0,\end{cases}
$$


which implies:

$$
\frac{\alpha_{0} \varphi_{k}}{\underline{\alpha}_{k+1}} \geq 1 \geq \frac{\alpha_{0} \varphi_{k}}{\bar{\alpha}_{k+1}}
$$

To prove these two inequalities, remember that $\phi=1-\frac{\sigma_{y}^{2}}{\sigma_{x}^{2}} \geq 0$ since $\sigma_{x}^{2}$ and $\sigma_{y}^{2}$ are assumed to be respectively the largest and smallest eigenvalues of $\Sigma_{\tilde{x} \tilde{y}}$. Indeed, $\frac{\alpha_{0} \varphi_{k}}{\underline{\alpha}_{k+1}} \geq 1$ is easily obtained from the positivity of all the terms $\left(\phi, \phi^{k}, \omega_{x}, \omega_{y}, p\right)$ involved in (A3). The second inequality comes from the following series of inequalities and from the binomial formula, still using positivity of the previous terms:

$$
\begin{aligned}
1+\phi^{k}\left(\frac{\omega_{x}(k+1)}{p}+\frac{\phi}{2}\right) & \leq 1+\frac{\omega_{x}(k+1)}{p}+\frac{\phi}{2} \\
& \leq 1+\frac{\left(\omega_{x}+\omega_{y}\right)(k+1)}{p}+\frac{\phi}{2} \\
& \leq\left(1+\frac{\left(\omega_{x}+\omega_{y}\right)}{p}+\frac{\phi}{2}\right)^{k+1} .
\end{aligned}
$$

Suppose $\underline{\alpha}_{k} \leq \alpha_{k} \leq \bar{\alpha}_{k}, \forall k \leq n$. Then:

$$
\sum_{k=0}^{n} \varphi_{k} \underline{\alpha}_{n-k} \leq \sum_{k=0}^{n} \varphi_{k} \alpha_{n-k} \leq \sum_{k=0}^{n} \varphi_{k} \bar{\alpha}_{n-k}
$$

Since $\underline{\alpha}_{n-k}=\frac{\alpha_{0} \underline{\alpha}_{n+1}}{\underline{\alpha}_{k+1}}$ and $\bar{\alpha}_{n-k}=\frac{\alpha_{0} \bar{\alpha}_{n+1}}{\bar{\alpha}_{k+1}}$, it comes that:

$$
\underline{\alpha}_{n+1} \sum_{k=0}^{n} \frac{\alpha_{0} \varphi_{k}}{\underline{\alpha}_{k+1}} \leq \sum_{k=0}^{n} \varphi_{k} \alpha_{n-k} \leq \bar{\alpha}_{n+1} \sum_{k=0}^{n} \frac{\alpha_{0} \varphi_{k}}{\bar{\alpha}_{k+1}} .
$$

Hence,

$$
\underline{\alpha}_{n+1}(n+1) \leq \sum_{i=0}^{n} \varphi_{i} \alpha_{n-i} \leq \bar{\alpha}_{n+1}(n+1),
$$

and the conclusion follows from (25).

It is then quite immediate to get the following result.

Corollary A.2. The coefficients $\left(\beta_{k}\right)_{k \geq 0}$ satisfy $\frac{\underline{\alpha}_{k}}{(k+1) !} \leq \beta_{k+1} \leq \frac{\bar{\alpha}_{k}}{(k+1) !}$.

Proof. Direct application of Proposition A.1 and Theorem III.2.

Upper and lower bounds for the truncation errors are now obtained based on the above minorant/majorant sequences. 
Proposition A.3. Let $\tilde{\mathcal{P}}_{n}(t)=\exp (-p t) \sum_{i=0}^{n-1} \frac{\alpha_{i}}{(i+1) !} t^{i+1}, \underline{\varepsilon}_{n}(t)=\exp (-p t) \sum_{i=n}^{\infty} \frac{\underline{\alpha}_{i}}{(i+1) !} t^{i+1}$ and $\bar{\varepsilon}_{n}(t)=e^{-p t} \sum_{i=n}^{\infty} \frac{\bar{\alpha}_{i}}{(i+1) !} t^{i+1}$. For each $n \geq 0$, one has

$$
\underline{\varepsilon}_{n}\left(R^{2}\right) \leq \mathcal{P}_{c}-\tilde{\mathcal{P}}_{n}\left(R^{2}\right) \leq \bar{\varepsilon}_{n}\left(R^{2}\right),
$$

with the particular case $\tilde{P}_{0}(t):=0$, for $n=0$.

Proof. From equation (26) and $\beta_{0}=0$, one gets: $\mathcal{P}_{c}-\tilde{\mathcal{P}}_{n}\left(R^{2}\right)=\exp \left(-p R^{2}\right) \sum_{k=n}^{\infty} \frac{\alpha_{k}}{(k+1) !} R^{2(k+1)}$. Apply then Corollary A.2.

To conclude the proof of the Proposition III.4, note that in general, for $\gamma>0$,

$$
\frac{1}{\gamma} \frac{(\gamma t)^{n+1}}{(n+1) !} \leq \sum_{i=n}^{\infty} \frac{\gamma^{i} t^{i+1}}{(i+1) !} \leq \frac{e^{\gamma t}}{\gamma} \frac{(\gamma t)^{n+1}}{(n+1) !}
$$

which gives straightforwardly $\underline{\varepsilon}_{n}\left(R^{2}\right) \geq l_{n}$ and $\bar{\varepsilon}_{n}\left(R^{2}\right) \leq u_{n}$, hence (30).

\section{Proof of Proposition III.5}

From equations (31), (32) and using the Stirling inequality [22] on $l_{n}$,

$$
\begin{aligned}
u_{n}-l_{n} & =l_{n}\left(\exp \left(p R^{2}\left(1+\frac{\phi}{2}+\frac{\omega_{x}+\omega_{y}}{p}\right)\right)\left(1+\frac{\phi}{2}+\frac{\omega_{x}+\omega_{y}}{p}\right)^{n}-1\right) \\
& <l_{n} \exp \left(p R^{2}\left(1+\frac{\phi}{2}+\frac{\omega_{x}+\omega_{y}}{p}\right)\right)\left(1+\frac{\phi}{2}+\frac{\omega_{x}+\omega_{y}}{p}\right)^{n} \\
& <\frac{\alpha_{0} \exp \left(-p R^{2}\right)\left(e p R^{2}\right)^{n+1}}{p \sqrt{2 \pi(n+1)}(n+1)^{n+1}} \exp \left(p R^{2}\left(1+\frac{\phi}{2}+\frac{\omega_{x}+\omega_{y}}{p}\right)\right)\left(1+\frac{\phi}{2}+\frac{\omega_{x}+\omega_{y}}{p}\right)^{n} \\
& <\left(\frac{e p R^{2}\left(1+\frac{\phi}{2}+\frac{\omega_{x}+\omega_{y}}{p}\right)}{n+1}\right)^{n+1} \frac{\alpha_{0} \exp \left(p R^{2}\left(\frac{\phi}{2}+\frac{\omega_{x}+\omega_{y}}{p}\right)\right)}{p \sqrt{2 \pi(n+1)}\left(1+\frac{\phi}{2}+\frac{\omega_{x}+\omega_{y}}{p}\right)} .
\end{aligned}
$$

For $n+1=N_{1}$ in the previous inequality, the obtained bound for the absolute error is:

$$
u_{N_{1}-1}-l_{N_{1}-1}<\left(\frac{1}{2}\right)^{N_{1}} \frac{\alpha_{0} \exp \left(p R^{2}\left(\frac{\phi}{2}+\frac{\omega_{x}+\omega_{y}}{p}\right)\right)}{p \sqrt{2 \pi N_{1}}\left(1+\frac{\phi}{2}+\frac{\omega_{x}+\omega_{y}}{p}\right)} .
$$

If this is not less than $\delta$, one has for all $N \geq N_{1}$ :

$$
u_{N-1}-l_{N-1} \leq\left(\frac{1}{2}\right)^{N} \frac{\alpha_{0} \exp \left(p R^{2}\left(\frac{\phi}{2}+\frac{\omega_{x}+\omega_{y}}{p}\right)\right)}{p \sqrt{2 \pi N_{1}}\left(1+\frac{\phi}{2}+\frac{\omega_{x}+\omega_{y}}{p}\right)} .
$$

Finally, one has (by construction):

$$
\left(\frac{1}{2}\right)^{N_{2}} \frac{\alpha_{0} \exp \left(p R^{2}\left(\frac{\phi}{2}+\frac{\omega_{x}+\omega_{y}}{p}\right)\right)}{p \sqrt{2 \pi N_{1}}\left(1+\frac{\phi}{2}+\frac{\omega_{x}+\omega_{y}}{p}\right)} \leq \delta,
$$

so, $n+1=\max \left\{N_{1}, N_{2}\right\}$ is suitable. 


\section{Proof of Proposition III.6}

In general, for $\gamma>0, \sum_{k=0}^{\infty} \frac{\gamma^{k} t^{k+1}}{(k+1) !}=\frac{e^{\gamma t}-1}{\gamma}$, hence:

$$
\sum_{k=0}^{\infty} \frac{\underline{\alpha}_{k} R^{2(k+1)}}{(k+1) !}=\alpha_{0} \frac{\exp \left(p R^{2}\right)-1}{p}
$$

and:

$$
\sum_{i=0}^{\infty} \frac{\bar{\alpha}_{i} R^{2(i+1)}}{(i+1) !}=\alpha_{0} \frac{\exp \left(p\left(1+\frac{\phi}{2}+\frac{\omega_{x}+\omega_{y}}{p}\right) R^{2}\right)-1}{p\left(1+\frac{\phi}{2}+\frac{\omega_{x}+\omega_{y}}{p}\right)}
$$

By multiplying the above expressions by $e^{-p R^{2}}$, one obtains: $l_{0}=\underline{\varepsilon}_{0}\left(R^{2}\right)=\alpha_{0} \frac{\exp \left(p R^{2}\right)-1}{p}$ and:

$$
\begin{gathered}
u_{0}=\bar{\varepsilon}_{0}\left(R^{2}\right)=\alpha_{0} \frac{\exp \left(p\left(\frac{\phi}{2}+\frac{\omega_{x}+\omega_{y}}{p}\right) R^{2}\right)-\exp \left(-p R^{2}\right)}{p\left(1+\frac{\phi}{2}+\frac{\omega_{x}+\omega_{y}}{p}\right)} \\
\underline{\varepsilon}_{n}\left(R^{2}\right)+\tilde{\mathcal{P}}_{n}\left(R^{2}\right)=e^{-p R^{2}}\left(\sum_{k=n}^{+\infty} \frac{\underline{\alpha}_{k}}{(k+1) !} R^{2(k+1)}+\sum_{k=0}^{n-1} \frac{\alpha_{k}}{(k+1) !} R^{2(k+1)}\right) \\
\geq e^{-p R^{2}} \sum_{k=0}^{\infty} \frac{\underline{\alpha}_{k} R^{2(k+1)}}{(k+1) !}=\underline{\varepsilon}_{0}\left(R^{2}\right) \\
\geq \alpha_{0} \frac{\exp \left(p R^{2}\right)-1}{p}
\end{gathered}
$$

Similarly, one gets: $\bar{\varepsilon}_{0}\left(R^{2}\right)+\tilde{\mathcal{P}}_{n}\left(R^{2}\right) \leq \bar{\varepsilon}_{0}\left(R^{2}\right)$.

Acknowledgments The Authors would like to thank Alexandre Falcoz from Airbus Defence and Space and the ANR FastRelax Project for the grants that partly supports this activity.

\section{References}

[1] K. T. Alfriend, M. R. Akella, J. Frisbee, F. L. Foster, D-J Lee, and M. Wilkins. Probability of collision error analysis. Journal of Space Debris, 1(1), 1999.

[2] M. R. Akella and K. T. Alfriend. Probability of collision between space objects. Journal of Guidance, Control and Dynamics, 23(5), September-October 2000.

[3] R. P. Patera. General method for calculating satellite conjunction probability. Journal of Guidance, Control and Dynamics, 24(4), July-August 2001.

[4] S. Alfano. A numerical implementation of spherical objet collision probability. Journal of Astronautical Sciences, 53(1), January-March 2005.

[5] F. K. Chan. Spacecraft Collision Probability. AIAA. The Aerospace Press, 2008.

[6] V.T. Coppola. Including Velocity Uncertainty in the Probability of Collision Between Space Objects. In AAS/AIAA Spaceflight Mechanics Meeting, number AAS 12-247, February 2012. 
[7] S. Alfano. Satellite conjunction monte carlo analysis. In Proceedings of AAS/AIAA Spaceflight Mechanics Meeting, number AAS 09-233, Savannah, Georgia, USA, February 2009.

[8] J. L. Foster and H. S. Estes. A parametric analysis of orbital debris collision probability and maneuver rate for space debris. NASA/JSC-25898, August 1992.

[9] S. Alfano. Aerospace support to space situation awareness. Applicationes Mathematicae, October 2002.

[10] F. K. Chan. Collision probability analyses for earth-orbiting satellites. Applicationes Mathematicae, 1997.

[11] J. B. Lasserre and E. S. Zeron. Solving a class of multivariate integration problems via laplace techniques. Applicationes Mathematicae, 2001.

[12] D. Zeilberger. A holonomic systems approach to special functions identities. Journal of Computational and Applied Mathematics, 32(3):321-368, 1990.

[13] B. Salvy. D-finiteness: Algorithms and applications. In Manuel Kauers, editor, ISSAC 2005: Proceedings of the 18th International Symposium on Symbolic and Algebraic Computation, Beijing, China, July 2427, 2005, pages 2-3. ACM Press, 2005. Abstract for an invited talk.

[14] A. Genz and F. Bretz. Computation of multivariate normal and $t$ probabilities, volume 45 . Springer, 2009.

[15] V.T. Coppola. Evaluating the Short Encounter Assumption of the Probability of Collision Formula. In AAS/AIAA Spaceflight Mechanics Meeting, number AAS 12-248, February 2012.

[16] A. Papoulis and S.U. Pillai. Probability, random variables, and stochastic processes. Tata McGraw-Hill Education, 2002.

[17] S. Chevillard and M. Mezzarobba. Multiple-Precision Evaluation of the Airy Ai Function with Reduced Cancellation. In A. Nannarelli, P.-M. Seidel, and P. T. P. Tang, editors, 21st IEEE SYMPOSIUM on Computer Arithmetic, pages 175-182, Los Alamitos, CA, Avril 2013. IEEE Computer Society.

[18] W. Gawronski, J. Müller, and M. Reinhard. Reduced cancellation in the evaluation of entire functions and applications to the error function. SIAM Journal on Numerical Analysis, 45(6):2564-2576, 2007.

[19] D. V. Widder. An introduction to transform theory. Academic Press New York, 1971.

[20] D.V. Widder. The Laplace transform. Princeton mathematical series. Princeton university press, 1946.

[21] P. Flajolet and R. Sedgewick. Analytic Combinatorics. Cambridge University Press, New York, NY, USA, 1 edition, 2009.

[22] W. Feller. An Introduction to Probability Theory and Its Applications, Volume 1,. John Wiley, 1957. 